\title{
Transit-time diodes and transistors with variable injection for generation and detection of $\mathrm{THz}$ radiation
}

\author{
Vladimir Vyurkov ${ }^{*}$, Igor Semenikhin, Konstantin Rudenko, and Vladimir Lukichev \\ Valiev Institute of Physics and Technology RAS, Nakhimovsky prosp. 34, Moscow, 117218, Russia
}

\begin{abstract}
The non-linear model of $\mathrm{THz}$ generation in variable injection transit-time structures coupled with antenna is proposed. The simulation demonstrates the crossover from the initial harmonic regime of selfexcitation to non-harmonic generation in the developed stage.
\end{abstract}

\section{Introduction}

In the range of terahertz frequencies, an abrupt depression in generation power of both optical and electronic devices occurs [1]. Therefore, the efficient $\mathrm{THz}$ sources and detectors predominantly working at room temperature are challenging.

In this paper, we continue the consideration of low-dimensional solid-state transit-time structures based on thin layers of silicon (2D) and an array of silicon nanowires (1D) for the generation and detection of terahertz radiation [2-7]. The advantage of such structures is that they can be fabricated using modern planar silicon technology. For the device implementation, the key technologies were developed and demonstrated: they allow the formation of low-dimensional structures coupled to antenna for the output of generated $\mathrm{THz}$ radiation and detection of $\mathrm{THz}$ radiation $[8,9]$. The schematic view of the transit-time generating cell inserted into antenna or waveguide with the typical dimensions for the $\mathrm{THz}$ frequencies of generation is drawn in Fig. 1.

A crucial property of the structure for generation with frequency $\omega$ is the presence of negative conductivity $\operatorname{Re} \sigma(\omega)$ (the negative real part of admittance $\operatorname{ReY}(\omega)$ ) in the linear regime):

$$
\operatorname{Re} \sigma(\omega)<0
$$

In this case, the system releases energy. It is clear that this originates only in a nonequilibrium system. In the transit-time diodes, the deviation from equilibrium is caused by the applied direct voltage. It is important to note that for transit-time diodes, the presence of negative differential conductivity in the static characteristics is not necessary, as is the case in the tunnel and resonance tunnel diodes [10].

* Corresponding author: vvyurkov@gmail.com 


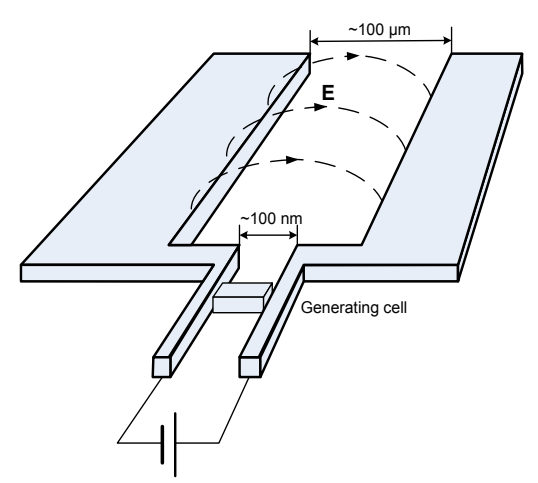

Fig. 1. Schematic view of the generating cell inserted into antenna or waveguide for $\mathrm{THz}$ frequencies of generation.

The delay of the current with respect to voltage results from a finite transit time. However, the complexity of the problem is caused by the fact that solely the presence of a delay cannot guarantee negative conductivity.

The first transit-time diodes which were used to generate $\mathrm{THz}$ radiation were electronic lamps. A ballistic (without scattering) current was implemented in them. One of the methods for generating $\mathrm{THz}$ generation using solid-state low-dimensional structures (namely, quantum ballistic wires based on A3B5 heterostructures) was proposed in 1994 [2]. It turned out that the negative conductivity in these diodes arises at terahertz frequencies. Modern equipment for fabrication of silicon chips also allows the creation of low-dimensional structures, such as thin films (2D) and arrays of nanowires (1D). Unfortunately, the negative conductivity in solid state transit-time diodes has a threshold with respect to the scattering rate, which is quite similar to the threshold of generation in optical devices. For the origination of negative conductivity in diodes with a drift-diffusion current (in the case of strong scattering), additional effects are required, such as impact ionization in avalanche-transit (IMPATT) diodes [11] or variable injection in barrierinjection transit-time (BARITT) diodes [12].

In present paper, we continue examining the variable injection similar to the BARITT effect, although the structure under consideration substantially differs from that in Ref. 12. As for the IMPATT effect, it leads to a decrease in efficiency and a much higher noise level due to the randomness of the impact ionization processes.

Worth noting, the transit-time phenomena are also important in solid-state plasmonic devices destined to $\mathrm{THz}$ generation and detection $[13,14]$.

The variable injection transit-time effect is illustrated in Fig. 2 for the diode (A) and transistor (B) structures. The essence is that the alternating voltage applied to source and drain in a diode or to floating gate and drain in a transistor influences on the barrier height, that is, on the injection to the channel. This effect is more pronounced in a transistor structure in comparison with the diode one.

\section{Non-linear model of generation in variable injection transit- time structures coupled with antenna}

Here we present the equations for simulation of generation in variable injection transit-time structures coupled with antenna in non-linear regime of generation. The drift current mode with the velocity saturation is taken into account. Hereafter the description belongs for electrons, although an elementary charge is supposed to be positive, that is $\mathrm{e}=|\mathrm{e}|$. 
In the transistor-like structure the gate and drain contacts are connected to antenna. Meanwhile, in the diode-like the antenna is attached to source and drain contacts, herewith some means should be undertaken to decouple direct current and alternating current circuits.

(A)
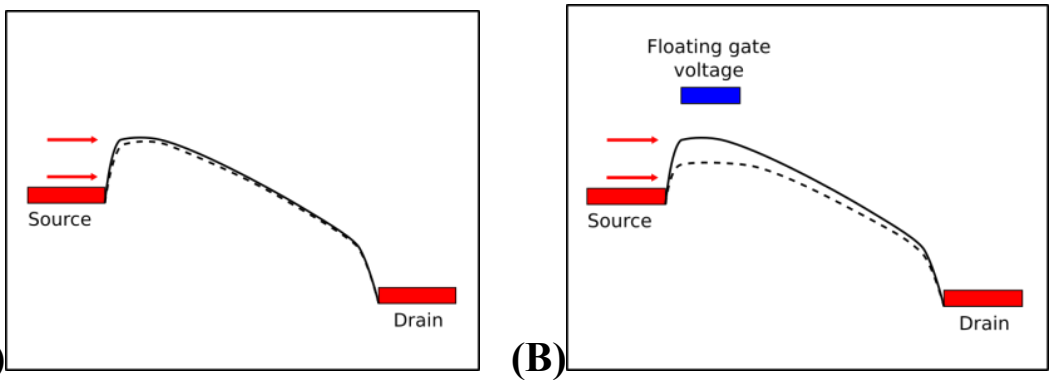

Fig. 2. Band diagrams of the structures. Weak variable injection effect in a diode structure (A) and strong effect in a transistor-like structure (B). The floating gate voltage controls the potential barrier height, hence, the current through the channel. In the case, the gate is connected with an antenna as well as the drain.

The direct positive voltage $V_{0}$ is applied to the transit-time element; this gives rise to a direct current $\mathrm{I}_{0}$. The alternating current $\mathrm{I}(\mathrm{t})$ and voltage $\mathrm{V}(\mathrm{t})$ are transmitted to antenna.

We use the continuity equation for the deviation of density $n(x, t)$ from its static value $n_{0}$ in the active element (diode or transistor channel elongated along the $\mathrm{x}$-axis):

$$
\frac{\partial n(x, t)}{\partial t}=-\operatorname{div}(p)=-\left(v_{0}+v(t)\right) \frac{\partial n(x, t)}{\partial x},
$$

where $p$ is the electron flux (opposite to the current), $v_{0}$ is the static velocity, $v(t)$ is the alternating velocity.

We take into account only the drift component of the current and omit the diffusion one. This requires a justification. Really, both components of current are

$$
j=e \mu n E+e D \nabla n,
$$

where the first term corresponds to the drift current, and the second one to the diffusion current. The gradient of the varying density $n$ could be roughly estimated as $\nabla n \approx n / l$, where $l$ is the channel length. Accounting for the Einstein relation

$$
\mu=\frac{e D}{k T}
$$

one comes at the condition when the drift current prevails:

$$
e V_{0}>k T
$$

This demand is definitely observed in experimental structures.

As for the space charge, the condition to neglect it was derived in Ref. 6, which is also valid for realistic systems.

The expressions for the static and alternating velocities in the drift approximation with velocity saturation look like

$$
v_{0}=\frac{v_{S} \operatorname{sign}\left(E_{0}\right)}{1+\frac{v_{S}}{\mu\left|E_{0}\right|}} \quad v_{0}+v(t)=\frac{v_{S} \operatorname{sign}\left(E_{0}+E(t)\right)}{1+\frac{v_{S}}{\mu\left|E_{0}+E(t)\right|}}
$$

where $E_{0}=V_{0} / l$ is the static electric field, $E(t)=V(t) / l$ is the alternating electric field, $l$ is the channel length, $\mu$ is the weak field mobility, the signum function $\operatorname{sign}(\mathrm{x})=\mathrm{x} /|\mathrm{x}|$ defines the 
velocity direction, which can alter only in strongly non-linear regime of generation, the saturation velocity in silicon equals $v_{S}=10^{7} \mathrm{~cm} / \mathrm{s}$, here the saturation is caused by the multiple emission of optical phonons.

The alternating current in the outer circuit (antenna) is provided by the ShockleyRameau theorem [15], which we exploited in previous papers:

$$
I(t)=-e n_{0} v(t)-\frac{e}{l}\left(v_{0}+v(t)\right) \int_{0}^{l} n(x, t) d x
$$

This technique is mandatory for the case when the period of oscillations is comparable with the transit time. The sense of the theorem lies in the fact that all charges moving in the channel contribute to the current in outer circuit. In Refs. 6-7 the complex conductivity of the transit-time element was calculated via the complex absorption in the channel. In fact, this approach can serve as a proof of the Shockley-Rameau theorem.

The outer circuit (antenna) is described by three parameters: capacity $\mathrm{C}$, inductance $\mathrm{L}$, and radiation resistance $\mathrm{R}$ connected sequentially.

The equations binding the alternating current with the voltage drop on corresponding components are as follows

$$
V_{L}(t)=L \frac{\partial I(t)}{\partial t}, \quad V_{C}(t)=\frac{\int_{0}^{t} I(\tau) d \tau}{C}, \quad V_{R}(t)=R \cdot I(t)
$$

According to the Kirchhoff's law the voltage drop in closed circuit is equal to zero:

$$
V(t)+V_{L}+V_{C}+V_{R}=0
$$

The boundary condition of varying injection from the left contact (the source or under the gate) is

$$
n(x=0, t)=n_{0}\left(e^{\frac{\alpha e V(t)}{k T}}-1\right)
$$

This formula describes the change in the potential barrier height caused by the alternating voltage $V(t)$, while the coefficient $\alpha$ characterizes the efficiency of that change. For transistor-like structures $\alpha$ is close to unity, at the same time, for diode-like structures the value of $\alpha$ is much smaller, for example, $\alpha=0.1$. Worth noting this condition is valid only for the positive sign of the total voltage $V_{0}+V(t)>0$. Otherwise, the boundary condition must be thrown over the opposite contact (the drain) where the injection originates:

$$
n(x=l, t)=n_{0}\left(e^{-\frac{\alpha e V(t)}{k T}}-1\right) .
$$

The initial condition for the perturbed density in the channel is

$$
n(x \neq 0, t=0)=\delta n(x),
$$

where $\delta n(x)$ is a random function of an appropriately small amplitude describing the starting density fluctuations in the channel. Those fluctuations are the basis for selfexcitation. One may also add a small voltage fluctuation to the initial conditions, for instance, $V(t=0)=0.01 V_{0}$.

Worth mentioning the velocity of injected particle is restricted by the thermal velocity $v(x=0) \leq v_{T}$.However, the saturation velocity in silicon is close to the thermal one at room temperature; therefore, we neglect this effect for simplification of the model.

The complete set of equation to be solved sequentially and iteratively is 


$$
\begin{aligned}
& \text { 1. } \frac{\partial n(x, t)}{\partial t}=-\frac{\mu}{l}\left(V_{0}+V(t)\right) \frac{\partial n(x, t)}{\partial x} \\
& \text { 2. } I(t)=\mu \frac{e}{l} n_{0} V(t)+\mu \frac{e}{l^{2}}\left(V_{0}+V(t)\right) \int_{0}^{l} n(x, t) d x \\
& \text { 3. } V(t)=-L \frac{d I(t)}{d t}-R I(t)-\frac{1}{C} \int_{0}^{t} I\left(t^{\prime}\right) d t^{\prime}
\end{aligned}
$$

Herewith the before mentioned boundary and initial conditions must be provided.

\section{Preliminary results of simulation}

Parameters of the structure are as follows: the channel length is $100 \mathrm{~nm}$, the mobility is 500 $\mathrm{cm} 2 / \mathrm{V} \mathrm{s}$ (for silicon), the direct voltage is $0.5 \mathrm{~V}$, and the temperature equals room temperature $\mathrm{kT}=0.025 \mathrm{eV}$. The constant velocity $v(t) \equiv v_{S}$ equal to the saturation velocity was used in the preliminary simulation; this is justified by a fairly high direct bias.

The impedance of antenna is

$$
Z(\omega)=R+i \omega L+\frac{1}{i \omega C},
$$

where the parameters $\mathrm{L}=310^{-12} \mathrm{H}, \mathrm{C}=310^{-15} \mathrm{~F}, \mathrm{R}=100 \mathrm{Ohm}$ are quite close to that in rigorous calculations regarding the experimental structures. The resonant frequency of antenna is equal to $1 \mathrm{THz}$ (the wave length in vacuum is equal to $300 \mu$ ).

Implicit second order finite difference method for time is used, while over the space coordinate the Chebyshev expansion is exploited.

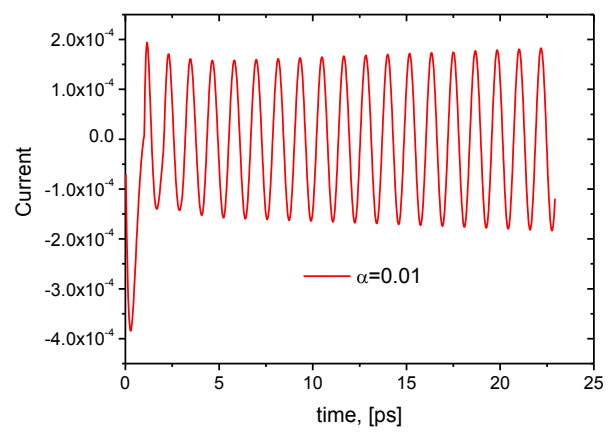

Fig. 3. The initial stage of current oscillations (a.u.) originating from fluctuations of density in the channel. Quite harmonic oscillations with slowly rising amplitude are visible.

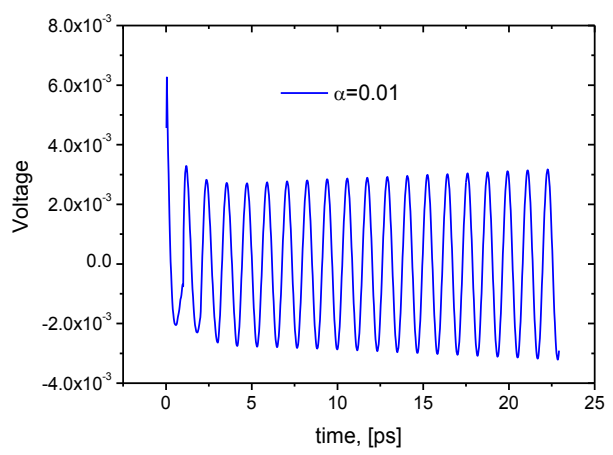

Fig. 4. The initial stage of voltage oscillations (a.u.) originating from fluctuations of density in the channel. Quite harmonic oscillations with slowly rising amplitude are visible. 


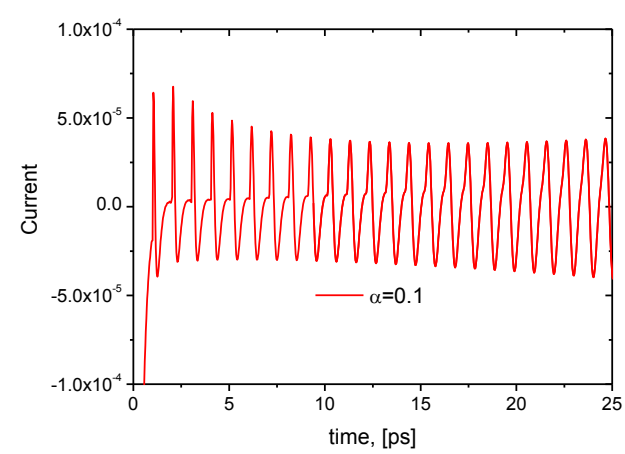

Fig. 5. The developed stage of current oscillations (a.u.) originating from fluctuations of density in the channel. Quite non-harmonic oscillations with slowly varying amplitude are visible.

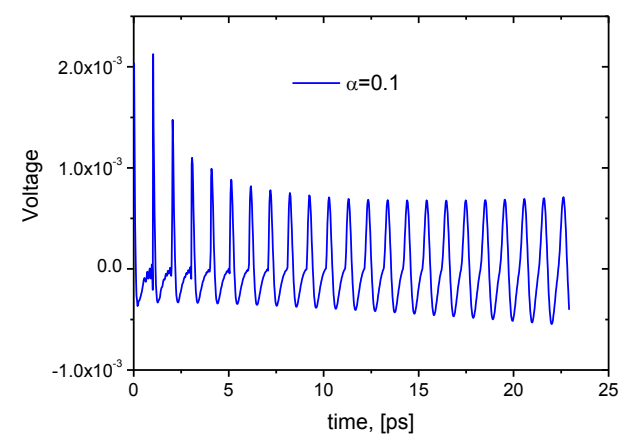

Fig. 6. The developed stage of voltage oscillations (a.u.) originating from fluctuations of density in the channel. Quite non-harmonic oscillations with slowly varying amplitude are visible.

Preliminary results of simulation are shown in Figs. 3-6. Unfortunately, up to date, we cannot still answer the main questions concerning the generation: what is its power and efficiency. To achieve an ultimate power and efficiency a thorough matching of a generating cell and antenna is required.

\section{Detection}

The $\mathrm{THz}$ radiation can be detected with the help of the same structures as used for generation [7]. This is very convenient from practical point of view. The detection is based on the rectifying properties of the structures under consideration. In fact, the change in the level of injection is caused by the alternating $\mathrm{THz}$ voltage applied to the structure from the antenna. Recently, the effect of rectification of $\mathrm{THz}$ radiation was experimentally observed in Ref. 16.

Worth noting, when the transit-time structure is close to the regime of generation at the frequency of incident $\mathrm{THz}$ radiation the responsivity of the detector could be substantially enhanced.

\section{Conclusions}

The non-linear model of $\mathrm{THz}$ generation in variable injection transit-time structures coupled with antenna is proposed. The simulation demonstrates the crossover from the initial harmonic regime of self-excitation to non-harmonic generation in the developed stage. The generation arises from self-excitation of thermal fluctuations existing in the 
channel. Surely, in simulation the amplitude of initial fluctuations was taken much higher than that in real systems to speed up the transition to developed oscillations which are the goal of current investigation.

\section{Acknowledgement}

The work was supported by Program no. 0066-2019-0004 of the Ministry of Science and Higher Education of Russia for Valiev Institute of Physics and Technology of RAS and also supported by RFBR, grants nos. 16-29-09510, 16-29-03402, 17-07-00994, 18-57-06001, 18-07-01145.

\section{References}

1. S.S. Dhillon, M.S. Vitiello, E.H. Linfield, E.H. et al., J. Phys. D: Appl. Phys. 50, 043001 (2017)

2. L. Fedichkin, V. V'yurkov, Appl. Phys. Lett. 64, 2535 (1994)

3. A. Pilgun, V. Vyurkov, L. Fedichkin, V. Borzdov, A. Orlikovsky, Int. Conf. Microand Nanoelectronics (ICMNE), Oct. 2012, Zvenigorod, Russia, Book of Abstracts, P109 (2012)

4. V. Vyurkov, D. Svintsov, A. Pilgun, A. Orlikovsky, Proc. 4th Russia-Japan-USA Symposium on Fundamental \& Applied Problems of Terahertz Devices \& Technologies (RJUS TeraTech-2015), Chernogolovka, Russia, 58 (2015)

5. R. Khabutdinov, I. Semenikhin, F. Davydov, V. Vyurkov, L. Fedichkin, M. Rudenko, A.V. Borzdov, V.M. Borzdov, V.M., Proc. SPIE 10224, 102240M (2016)

6. V.V. Vyurkov, R.R. Khabutdinov, A.B. Nemtsov, I.A. Semenikhin, M.K. Rudenko, K.V. Rudenko, V.F. Lukichev, Russian Microelectronics 47, 290 (2018)

7. V. Vyurkov, A. Miakonkikh, A. Rogozhin, M. Rudenko, K. Rudenko, V. Lukichev,Proc. SPIE 11022, 1102202 (2019)

8. A.V. Miakonkikh, A.A. Tatarintsev, A.E. Rogozhin, K.V. Rudenko, Proc. SPIE 10224, 102241V (2016)

9. A.V. Miakonkikh, N.A. Orlikovskiy, A.E. Rogozhin, K.V. Rudenko, A.A. Tatarintsev, Russian Microelectronics 47, 157 (2018)

10. M. Asada, S. Suzuki, N. Kishimoto, Jap. J. Appl. Phys. 47, 4375 (2008)

11. A.S. Tager, Sov. Phys. Usp. 9, 892 (1967)

12. D.J. Coleman jr., Jr., J. Appl. Phys. 43, 1812 (1972)

13. V. Ryzhii, A. Satou, M.S. Shur, Phys. Status Solidi A 202, R113 (2005)

14. A.S. Petrov, D. Svintsov, M. Rudenko, V. Ryzhii, M.S. Shur, M.S., Int. J. High Speed Electron. Syst. 25, 1640015 (2016)

15. W. Shockley W., J. Appl. Phys. 9, 635 (1938)

16. A. Boukhayma, A. Dupret, J.-P. Rostaing, C. Enz, C., Sensors (Basel) 16, 325 (2016) 